\title{
Dynamic Elements at Both Cytoplasmically and Extracellularly Facing Sides of the UapA Transporter Selectively Control the Accessibility of Substrates to Their Translocation Pathway
}

\author{
Vasiliki Kosti, loannis Papageorgiou and George Diallinas*
}

\author{
Department of Botany, \\ Faculty of Biology, \\ University of Athens, \\ Panepistimioupolis, \\ Athens 15781, Greece
}

Received 17 December 2009; received in revised form

17 February 2010;

accepted 19 February 2010

Available online

25 February 2010

\begin{abstract}
In the UapA uric acid-xanthine permease of Aspergillus nidulans, subtle interactions between key residues of the putative substrate binding pocket, located in the TMS8-TMS9 loop (where TMS is transmembrane segment), and a specificity filter, implicating residues in TMS12 and the TMS1-TMS2 loop, are critical for function and specificity. By using a strain lacking all transporters involved in adenine uptake $(\Delta a z g A \Delta f c y B \Delta u a p C)$ and carrying a mutation that partially inactivates the UapA specificity filter (F528S), we obtained 28 mutants capable of UapA-mediated growth on adenine. Seventy-two percent of mutants concern replacements of a single residue, R481, in the putative cytoplasmic loop TMS10-TMS11. Five missense mutations are located in TMS9, in TMS10 or in loops TMS1TMS2 and TMS8-TMS9. Mutations in the latter loops concern residues previously shown to enlarge UapA specificity (Q113L) or to be part of a motif involved in substrate binding (F406Y). In all mutants, the ability of UapA to transport its physiological substrates remains intact, whereas the increased capacity for transport of adenine and other purines seems to be due to the elimination of elements that hinder the translocation of nonphysiological substrates through UapA, rather than to an increase in relevant binding affinities. The additive effects of most novel mutations with F528S and allele-specific interactions of mutation R481G (TMS10TMS11 loop) with Q113L (TMS1-TMS2 loop) or T526M (TMS12) establish specific interdomain synergy as a critical determinant for substrate selection. Our results strongly suggest that distinct domains at both sides of UapA act as selective dynamic gates controlling substrate access to their translocation pathway.
\end{abstract}

(c) 2010 Elsevier Ltd. All rights reserved.

Keywords: Aspergillus nidulans; nucleobase ascorbate transporter (NAT) family; structure-function relationships; uric acid tions with UapC (a kinetically distinguishable UapA paralogue $)^{4}$ and randomly selected or rationally designed mutations, combined with green fluorescent protein (GFP)-based fluorescence microscopy and detailed kinetic analyses of functional mutants using a collection of purine analogues, have led to important conclusions concerning structure-function-specificity relationships in this permease. ${ }^{5-10}$ Of primary importance are the findings strongly supporting that the long amphiphilic loop linking transmembrane segments 8 and 9 (TMS8-TMS9) contains basic elements of the substrate translocation pathway. $5,7,10$ These are reflected in four irreplaceable amino acid residues (E356, D388, Q408, N409) and several other residues affecting 
UapA function, substrate affinity or specificity (A363, E371, R373, G411, T416, R417). Several of these critical residues (Q408, N409, G411, T416, R417) define the so-called NAT (nucleobase ascorbate transporter) signature motif (residues 406-417) present in all UapA homologues constituting the NAT family. ${ }^{2-4}$ Kinetic evidence has strongly suggested that this motif interacts with the imidazole moiety of xanthine, uric acid or other UapA ligands. ${ }^{7,11}$ In this interaction, Q408 could make a direct $\mathrm{H}$-bond with position $\mathrm{N} 9$ or $\mathrm{C} 8=\mathrm{O}$ of xanthine or uric acid, respectively, whereas N409 seems to be a dynamic element absolutely necessary for molecular movements associated with transport. Finally, G411 and T416 are important for narrowing the specificity of UapA to xanthine and uric acid, whereas R417 increases the affinity of UapA specifically for uric acid. ${ }^{7,11}$ An absolutely necessary functional role has also been assigned to E356, whereas the absolute need for D388 seems to be associated with a structural role rather than a functional one. ${ }^{10}$

A second important conclusion concerns three residues enlarging UapA specificity without affecting its kinetic behavior toward physiological substrates, uric acid and xanthine. These are T526 and F528 in TMS12 and Q113 in the short putative extracytoplasmic loop linking TMS1 and TMS2 ${ }^{6,9,10}$ (see Fig. 1a). A weaker but similar effect on UapA specificity was also obtained with several rationally designed mutations along TMS1, ${ }^{10}$ some of which can also affect UapA function and turnover. ${ }^{12}$ Allele-specific interactions and kinetic evidence have strongly suggested that differential positioning of TMS1 and TMS12 with respect to the TMS8TMS9 region acts as a dynamic filter to allow or restrict access of different purines to the substrate binding pocket (discussed also later). ${ }^{10}$

Interestingly, UapA is probably unique among NAT homologues of known function with respect to its high affinity and capacity for uric acid transport. ${ }^{2,3}$ All other NAT members of prokaryotes and fungi show high affinity for either xanthine or uracil $(<10 \mu \mathrm{M})$ but only moderate $(>50 \mu \mathrm{M})$ or no affinity for uric acid. The only other NAT member showing high affinity for uric acid is the maize Lpe1 carrier. $^{92}$ Interestingly, known mammalian NAT homologues, which are $\mathrm{Na}^{+}$rather than $\mathrm{H}^{+}$symporters, are specific either for nucleobases (uracil and xanthine mostly) or for L-ascorbate. ${ }^{13,14}$ However, none of the characterized bacterial, plant or fungal NATs can transport ascorbate. ${ }^{2,3}$ The unique efficiency for uric acid recognition and transport of UapA seems to depend on specific synergistic interdomain interactions that cannot be reproduced in other NAT members or in chimeric molecules where TMS1, TMS12 or TMS8-TMS9 of UapA has been replaced by that of homologous carriers from fungi (UapC), ${ }^{9}$ bacteria (YgfO) ${ }^{9}$ or mammals (SVCT2) (S. Amillis and G. Diallinas, unpublished results).

Analogous rationally designed mutations and the substituted-cysteine accessibility method in the
Escherichia coli xanthine permease $\mathrm{YgfO}$, a prototype bacterial member of the NAT family, have led to very similar conclusions with respect to the functional role of the TMS8-TMS9 region and in particular the NAT signature motif and the four functionally irreplaceable amino acid residues. ${ }^{15-18}$ However, in line with the finding that fine interdomain interactions in NAT carriers are proteinspecific and critical for specificity, none of the YgfO mutations that correspond to the UapA specificity mutations has led to a carrier that can efficiently recognize and transport a purine other than xanthine.

In this work, we used a novel two-step genetic approach to isolate UapA versions that can efficiently transport adenine. Our results show that we obtained mutations defining novel elements of the UapA specificity/selectivity filter, including a putative cytoplasm-facing loop. These results are discussed with respect to the importance of interdomain synergy and the role of loops as dynamic gates at both sides of the transporter in substrate selection.

\section{Results}

\section{Rationale of a novel genetic screen for UapA mutants able to transport adenine}

Based on detailed kinetic analyses of the wildtype, single and double mutants, we have proposed a model that rationalizes UapA-substrate interactions. 2,30,11 In brief, in the wild type, uric acid or xanthine enters into the putative substrate binding pocket, an essential part of which is made by TMS8-TMS9, and binds to specific residues with high affinity through two strong $\mathrm{H}$-bonds implicating $\mathrm{N} 1-\mathrm{H}$ and $\mathrm{N} 9$ (xanthine) or $\mathrm{N} 1-\mathrm{H}$ and $\mathrm{C} 8=\mathrm{O}$ (uric acid) and a weaker bond involving $\mathrm{C} 2=\mathrm{O}$. Purines, other than xanthine or uric acid, seem to be excluded from the wild-type substrate binding pocket due to steric and/or repulsive forces exerted by the positioning of specific residues in TMS1, in the TMS1-TMS2 loop and in TMS12, which thus collectively act as a molecular filter. All previously identified UapA residues that affect substrate binding and transport, and especially those mapping in the NAT signature motif, seem to be involved in interactions with the imidazole moiety of purines. ${ }^{7,9,10}$ We still ignore which part of UapA, or any other NAT member, interacts with the pyrimidine moiety of purines.

To approach this puzzle, we thought of selecting UapA mutants that would transport adenine, a purine that has a distinct pyrimidine moiety compared with the natural substrates of UapA. Considering the proposed UapA-substrate interaction model, two genetic modifications on the permease molecule should be satisfied to acquire high affinity for adenine: (1) the substrate binding pocket should be accessible to adenine and (2) an appropriately positioned residue should interact 
(a)
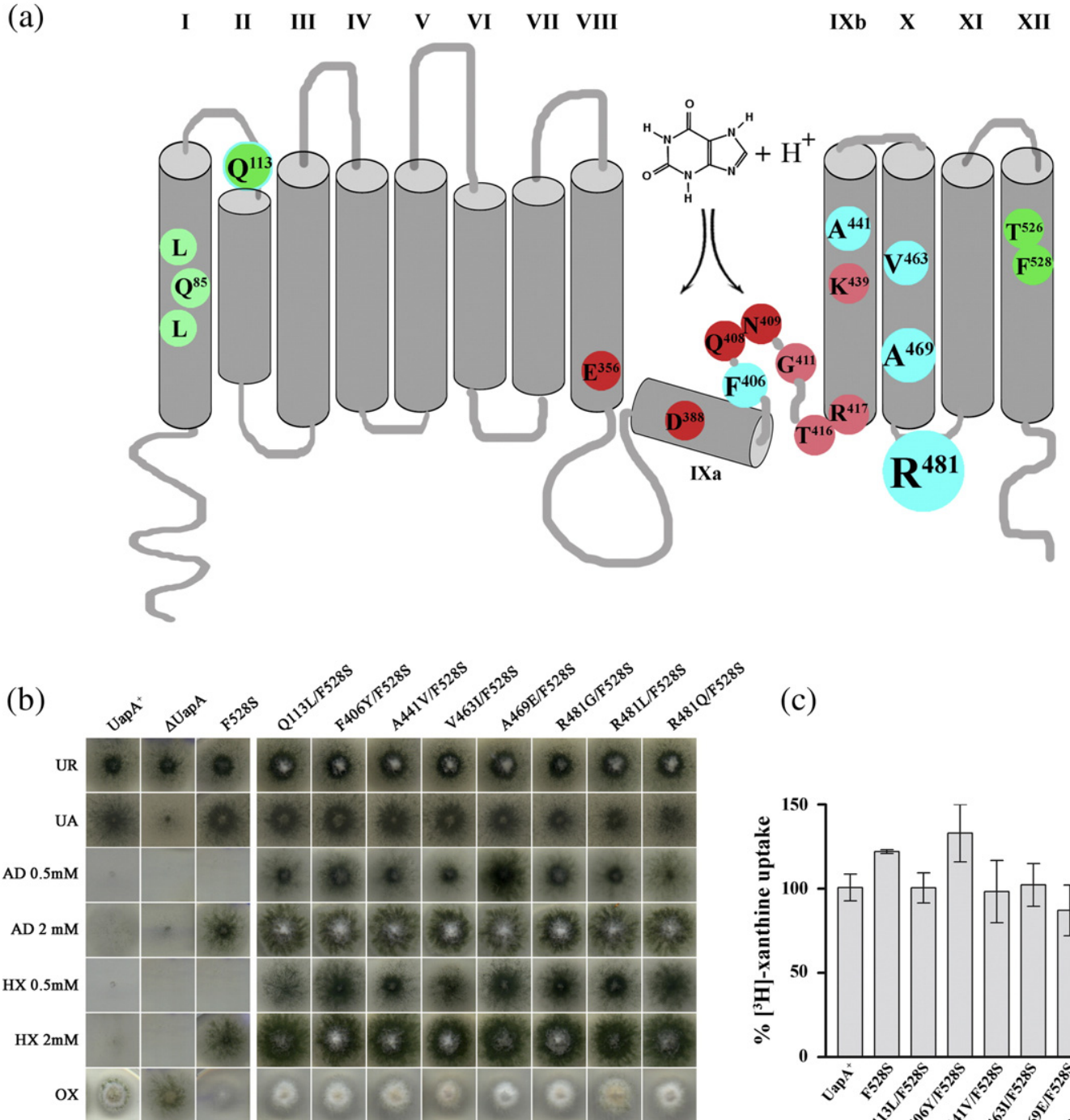

(c)

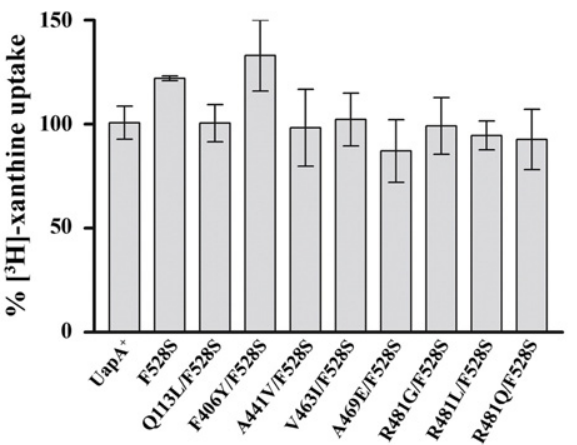

Fig. 1. (a) Topology of UapA and specificity mutations. Previously characterized residues affecting UapA function and/or specificity are marked in red (absolutely necessary for function), pink (affecting transport kinetics and specificity) or green (affecting specificity but not transport kinetics for physiological substrates). Lighter green signifies milder effects on UapA specificity. Residues in the TMS8-TMS9 loop (in red) are elements of the putative substrate binding site of UapA, whereas those in TMS1 and TMS12 (in green) are elements of a specificity filter restricting access of purines other than uric acid and xanthine to the bona fide substrate site. Mutations isolated in this work are highlighted in blue. R481 is emphasized in size to denote the high frequency $(72 \%)$ of mutations concerning this residue. Latin numbers denote putative TMSs. (b) Growth phenotypes of strains expressing wild-type UapA ${ }^{+}$or UapA-F528S (F528S) on purines as sole nitrogen sources (left panel). A strain with total deletion of the uapA locus ( $\Delta$ UapA) is also shown as a negative control. Growth on urea (UR, $5 \mathrm{mM}$ ) is used as a control. Purines tested as nitrogen sources include uric acid (UA, $0.5 \mathrm{mM}$ ), adenine (AD, 0.5 and $2 \mathrm{mM}$ ) and hypoxanthine (HX, 0.5 and $2 \mathrm{mM})$. OX is oxypurinol $(100 \mu \mathrm{M})$, a toxic analogue of uric acid/xanthine that is taken up by UapA. Growth on xanthine has also been tested, and results are identical with those of UA (not shown). Growth tests were performed at $37^{\circ} \mathrm{C}$ and $\mathrm{pH} 6.8$, which are the standard growth conditions of $A$. nidulans in the laboratory. Similar results were obtained at $25^{\circ} \mathrm{C}$. Note that UapA expression mediates normal growth on UA (normal colony growth rate and conidiation typically observed in minimal medium) and partial sensitivity to OX (reduced colony diameter and lack of conidiation) but cannot support growth on AD or HX. Expression of UapA-F528S leads to similar $\mathrm{UapA}^{+}$growth on UA but also confers the ability for growth on high concentrations (2 mM) of AD or HX and oversensitivity to OX. Strain UapA-F528S was used to select for mutants growing on low AD (0.5 mM) concentrations. The identity, frequency and topology of mutations isolated are shown in Table 1 and in (a), whereas growth phenotypes of corresponding mutants on purines are shown in the right panel of $(b)$. Notice that all new mutants isolated differ from the original strain (UapA-F528S) used for their selection in their ability to grow stronger on AD or HX, particularly evident at low concentrations of these purines $(0.5 \mathrm{mM})$. (c) Relative ${ }^{3} \mathrm{H}$-labelled xanthine transport rates of the new mutants expressed as percentage of initial uptake rates $(V)$ compared with the wild-type $\left(\mathrm{UapA}^{+}\right)$rate. Standard deviations in all experiments were always $<20 \%$ of the mean values shown. 
with the non-protonated N1 atom or the amide $\left(-\mathrm{NH}_{2}\right)$ groups of $\mathrm{C} 6$ or $\mathrm{C} 2$. The first condition is already satisfied by several previously isolated mutations, such as those concerning Q113 (TMS1TMS2 loop), T526 or F528 (TMS12). ${ }^{18}$ These mutants grow equally well with uap $A^{+}$strains on standard uric acid and xanthine concentrations $(>0.3 \mathrm{mM})$, but their growth on adenine or hypoxanthine necessitates higher concentrations $(>1-2 \mathrm{mM})$ because the relevant binding affinities are very low (see Fig. 1b, left panel). Using one of these mutants (UapA-F528S), we wanted to obtain second-site mutations that will allow improved growth on lower concentrations $(0.5 \mathrm{mM})$ of adenine. An additional prerequisite for this scheme was that the original mutant strain used should lack all other carriers that are able to transport adenine. This strain was made by introducing, through transformation, the uapA-F528S allele in a genetic background lacking all endogenous transporters mediating adenine transport ( $\Delta$ uapC $\Delta a z g A$ $\Delta f(y B)^{19}$ (see Materials and Methods). UapA-F528S was selected among all available specificity mutants that perform very low-affinity adenine transport because it was the only one that in the $\Delta u a p C \Delta a z g A$ $\Delta f c y B$ background showed practically no growth on adenine at concentrations $<1 \mathrm{mM}$ (see Fig. 1b). UV mutagenesis of the UapA-F528S/ $\Delta a z g A \quad \Delta f c y B$ $\Delta$ uapC strain and selection for growth on $0.5 \mathrm{mM}$ adenine as sole nitrogen source led to the isolation of 65 mutants.

\section{Molecular and functional characterization of UapA mutants}

After purification, all mutants were tested on various purines as nitrogen sources. All were capable of growth in all purines, at both 25 and $37^{\circ} \mathrm{C}$ (not shown). The uapA open reading frame (ORF) of 28 of them was obtained by PCR and sequenced. In all cases, a single base substitution, besides the original F528S mutation, was detected within the uapA ORF (Table 1). The 28 mutations corresponded to 8 amino acid substitutions (Fig. $1 \mathrm{a}$ and Table 1). The great majority (71.4\%) involved substitutions of R481, a residue located in the putative short loop linking TMS10 and TMS11 (Fig. 1a). Five mutations (17.1\%) involved two residues, V463 and A469, located in TMS10. The remaining three were obtained once and involved residues Q113 (in the TMS1-TMS2 loop), F406 (in the NAT motif in TMS8-TMS9 loop) and A441 (in TMS9). Interestingly, mutation Q113L was also obtained previously as a mutation conferring UapA-mediated growth on $2 \mathrm{mM}$ adenine. Figure $1 \mathrm{~b}$ shows the growth phenotypes of the 8 mutants obtained. For most of them, growth on adenine or hypoxanthine was stronger at $2 \mathrm{mM}$ compared with that at $0.5 \mathrm{mM}$, whereas growth on uric acid and xanthine was identical in the range $0.5-2.0 \mathrm{mM}$. A possible exception was mutant A469E/F528S, which seemed to grow significantly better than all other mutants at
Table 1. Topology and frequency of second-site specificity mutations obtained in mutant UapA-F528S

\begin{tabular}{lccc}
\hline $\begin{array}{l}\text { Amino acid } \\
\text { substitution }\end{array}$ & $\begin{array}{c}\text { Topology } \\
\text { in UapA }\end{array}$ & $\begin{array}{c}\text { Nucleotide } \\
\text { change }\end{array}$ & $\begin{array}{c}\text { Number } \\
\text { obtained }\end{array}$ \\
\hline Q113L & TMS1-TMS2 loop & CAG $\rightarrow$ CTG & 1 \\
F406Y & TMS8-TMS9 loop & TTT $\rightarrow$ TAT & 1 \\
A441V & TMS9 & GCT $\rightarrow$ GTT & 1 \\
V463I & TMS10 & GTC $\rightarrow$ ATC & 2 \\
A469E & TMS10 & GCG $\rightarrow$ GAG & 3 \\
R481G & TMS10-TMS11 loop & CGG $\rightarrow$ GGG & 1 \\
R481Q & TMS10-TMS11 loop & CGG $\rightarrow$ CAG & 14 \\
R481L & TMS10-TMS11 loop & CGG $\rightarrow$ CTG & 5 \\
Total & & CGG $\rightarrow$ CTA & \\
\hline
\end{tabular}

TMS stands for predicted TMS of the UapA. Nucleotide changes are highlighted in bold.

$0.5 \mathrm{mM}$ adenine. These observations suggested that the mutants isolated might still have relatively low affinity for purines, other than the physiological substrates of UapA.

Uptake studies showed that all mutants have a very similar capacity for ${ }^{3} \mathrm{H}$-labelled xanthine influx compared with the $\mathrm{UapA}^{+}$wild-type control (Fig. 1c). These activities were dependent on the $\mathrm{H}^{+}$gradient of the plasma membrane, as evidenced from the transport inhibition by $\mathrm{H}^{+}$gradient uncouplers (not shown). However, no measurable uptake of radiolabelled hypoxanthine was obtained (not shown). This apparent paradox of no detectable hypoxanthine uptake in mutants able to grow on this purine has also been obtained with several previously isolated mutants. These mutants were shown to exhibit very low hypoxanthine or adenine binding affinities $\left(K_{\mathrm{i}}>2 \mathrm{mM}\right)$, and thus it is technically impossible to detect hypoxanthine or adenine uptake in standard assays performed with concentrations of $0.2-2.0 \mu \mathrm{M}$ radiolabelled purines. ${ }^{9,10}$ Similarly, the very low affinity of the novel mutants for purines other than uric acid or xanthine was confirmed by measuring $K_{i}$ values of purines, pyrimidines and analogues (see below). Thus, the transport profile of all new isolates is typical of UapA mutants with relaxed specificity, exhibiting high-capacity transport but very low binding affinities for purines other than xanthine or uric acid.

\section{Substrate binding profile of UapA mutants}

A detailed kinetic profile, reflected in $K_{\mathrm{m} / \mathrm{i}}$ values, was established as previously described (see Materials and Methods) for all eight new mutations (Table 2). All mutations affected little the affinity of UapA for uric acid or xanthine $\left(K_{\mathrm{m} / \mathrm{i}}\right.$ values of 0.8-15 $\mu \mathrm{M})$ compared with either the wild-type protein or the original UapA-F528S mutant $\left(K_{\mathrm{m} / \mathrm{I}}\right.$ of 3-11 $\mu \mathrm{M})$. Mutations of R481 had the strongest effect as reflected in $K_{\mathrm{m} / \mathrm{i}}$ values for uric acid or xanthine reduced by 2 - to 12 -fold. Several mutants also showed a minor increase (up to 4-fold) in affinity for adenine, hypoxanthine or guanine compared with the original F528S strain, but their actual 
Table 2. Substrate binding profile of specificity mutants obtained in F528S genetic background

\begin{tabular}{|c|c|c|c|c|c|c|c|c|c|c|}
\hline \multirow[b]{2}{*}{ Substrate/ligand } & \multicolumn{10}{|c|}{$K_{\mathrm{m} / \mathrm{i}}(\mu \mathrm{M})$} \\
\hline & $\mathrm{UapA}^{+}$ & F528S & $\begin{array}{c}\text { Q113L/ } \\
\text { F528S }\end{array}$ & $\begin{array}{c}\text { F406Y/ } \\
\text { F528S }\end{array}$ & $\begin{array}{c}\text { A441V/ } \\
\text { F528S }\end{array}$ & $\begin{array}{c}\text { V463I/ } \\
\text { F528S }\end{array}$ & $\begin{array}{c}\text { A469E/ } \\
\text { F528S }\end{array}$ & $\begin{array}{c}\text { R481G/ } \\
\text { F528S }\end{array}$ & $\begin{array}{c}\text { R481L/ } \\
\text { F528S }\end{array}$ & $\begin{array}{c}\text { R481Q/ } \\
\text { F528S }\end{array}$ \\
\hline Xanthine & 8 & 3 & 3 & 1.3 & 6 & 13 & 1.5 & 1.6 & 1.0 & 0.8 \\
\hline Uric acid & 7 & 11 & 4 & 6 & 15 & 12 & 2 & 0.9 & 1.4 & 1.3 \\
\hline Adenine & NI & NI & $\sim 2000$ & $\sim 2000$ & $\sim 1800$ & NI & $\sim 2000$ & NI & NI & NI \\
\hline Hypoxanthine & NI & $\sim 2000$ & $\sim 1500$ & $\sim 2000$ & $\sim 1100$ & NI & $\sim 1800$ & $\sim 2000$ & $\sim 1600$ & $\sim 1600$ \\
\hline Guanine & NI & $\sim 2000$ & 982 & $\sim 1000$ & 500 & NI & 500 & NI & NI & NI \\
\hline Uracil & NI & $\sim 2000$ & 77 & 322 & 123 & 386 & 127 & 75 & 113 & 35 \\
\hline 1-Methyl-xanthine & 280 & 420 & 820 & $\sim 1000$ & 88 & $\sim 1000$ & 300 & 247 & 851 & 151 \\
\hline 2-Thio-xanthine & 63 & 63 & 16 & 59 & 20 & 38 & 100 & 10 & 12 & 8 \\
\hline 3-Methyl-xanthine & 28 & 24 & 5 & 7 & 10 & 57 & 30 & 14 & 3 & 4 \\
\hline 6-Thio-xanthine & 350 & 92 & 9 & 50 & 22 & 56 & 20 & 13 & 4 & 8 \\
\hline 7-Methyl-xanthine & NI & $\sim 1000$ & 46 & 274 & 92 & 210 & 88 & 89 & 115 & 48 \\
\hline 8-Methyl-xanthine & 100 & 45 & 8 & 9 & 10 & 33 & 10 & 9 & 7 & 4 \\
\hline 9-Methyl-xanthine & 200 & 345 & 452 & 302 & 662 & $\sim 1000$ & $\sim 1000$ & $\sim 1000$ & NI & 161 \\
\hline Oxypurinol & 100 & 45 & 6 & 25 & 9 & 31 & 22 & 3 & 3 & 10 \\
\hline
\end{tabular}

$K_{\mathrm{m} / \mathrm{i}}$ values were determined as described in Materials and Methods. NI indicates no inhibition (90\%-100\% uptake). The ' ' symbol stands for values $>1000 \mu \mathrm{M}$ that were approximately estimated. Results are averages of at least three independent experiments with three replicates for each concentration point. Standard deviation was $<20 \%$.

affinities for these purines were still very low $\left(K_{\mathrm{m} / \mathrm{i}}>500 \mu \mathrm{M}\right)$. This was in agreement with growth tests showing that full growth on adenine or hypoxanthine was only achieved at $2 \mathrm{mM}$. These results show that the mutants isolated have an increased capacity for adenine or hypoxanthine accumulation and most probably for several other nucleobases, which, however, is not due to a significant increase in corresponding binding affinities. This is highlighted mostly in mutants of R481, which show no or very little measurable affinity for these purines despite growing strongly on adenine or hypoxanthine (Table 2).

Interestingly, all mutants showed a tendency of increased binding affinities for uracil, oxypurinol and several xanthine analogues with bulky substitutions at positions 2, 3, 7 and 8. None of the mutations, however, increases the affinity of UapA for analogues substituted at positions N1 and N9, which are proposed to be absolutely critical for direct interactions with the substrate binding site ${ }^{10}$ (Table 2). Among all mutants, those concerning R481 showed the highest increase in UapA affinities for uracil, oxypurinol and bulky xanthine analogues. This picture not only supported the proposed model on the importance of the different positions in the purine ring for binding to the substrate pocket but also resembled the effect of previously isolated specificity mutations mapping in the TMS1-TMS2 loop and in TMS12. 2,3,9,10

\section{Additive effects of novel mutations and F528S}

We constructed by genetic transformation strains expressing the novel uap $A$ mutation alleles in the absence of F528S. In all new strains, uapA alleles were expressed from their endogenous promoter from plasmids integrated in single or multiple copies (see Materials and Methods). Figure 2 and Table 3 show the analysis of these strains. In the absence of F528S, all mutants, in single- or multiplecopy plasmid versions, showed nearly wild-type growth on uric acid or xanthine, similar to that also obtained in the presence of F528S. In contrast, growth on other purines was significantly different from that obtained in the presence of F528S. In particular, all mutants arising from single-copy plasmid integration, besides $\mathrm{A} 441 \mathrm{~V}$, retained very little or even lost the ability for growth on $0.5 \mathrm{mM}$ adenine or hypoxanthine. In some mutants, but not those concerning R481 and A441, growth on adenine or hypoxanthine was stronger in multiple-copy plasmid mutants. Most single-copy plasmid mutants, besides F406Y and V463I, could grow well, similar to F528S, at elevated adenine or hypoxanthine concentrations $(2 \mathrm{mM})$. The F406Y and V463I mutants were also more resistant to oxypurinol, resembling a control strain lacking UapA activity.

Uptake studies showed that none of the mutations significantly affected UapA-mediated, $\mathrm{H}^{+}$-dependent transport of ${ }^{3} \mathrm{H}$-labelled xanthine (Fig. $2 b$ and not shown). Furthermore, no measurable uptake of radiolabelled hypoxanthine was obtained (not shown), predicting that all single mutants exhibit very low binding affinities for purines other than their natural substrates. This was confirmed by directly measuring $K_{\mathrm{i}}$ values of purines, pyrimidines and analogues. Table 3 shows the substrate binding profile of the novel mutants established in the absence of F528S. It was clear that this is different from the one obtained in the genetic background of the F528S mutation. Affinities for xanthine and uric acid were close to that of the wild-type protein (2- to 3-fold differences), with the exception of V463I and R481Q, which have $K_{\mathrm{m}}$ values for xanthine reduced by 6- and 9-fold, respectively, and A469E, which has a $K_{i}$ value for uric acid increased by 8 -fold. A very moderate increase in the affinity for adenine was obtained only for some mutants (V463I, A469E), whereas there is a stronger and more general trend 
(a)

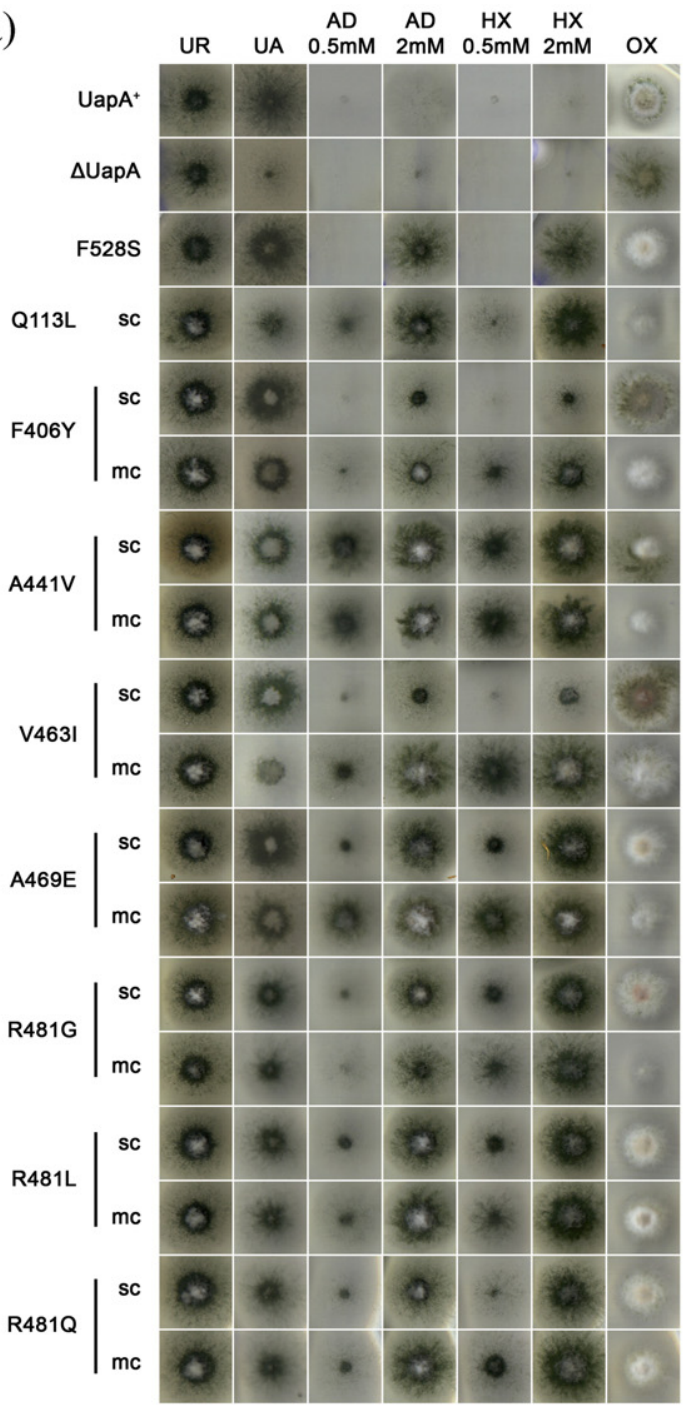

(b)

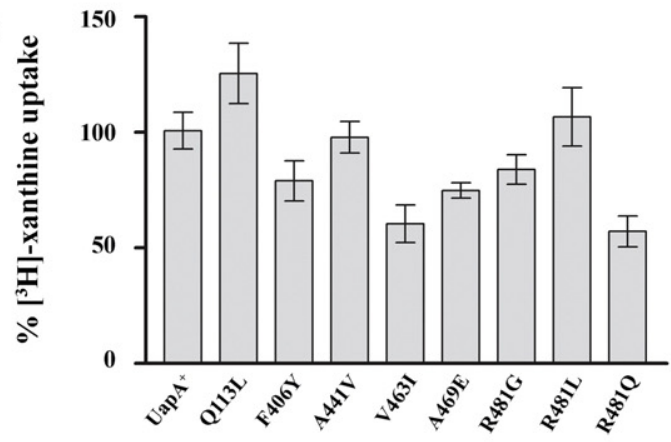

Fig. 2. (a) Growth tests of the novel mutations in the absence of the original substitution on different purines as nitrogen sources. Control strains and growth conditions are described in Fig. 1b. (b) Relative ${ }^{3} \mathrm{H}$-labelled xanthine transport rates of single-copy novel mutations expressed as percentage of initial uptake rates $(V)$ compared with the wild-type $\left(\mathrm{UapA}^{+}\right)$rate, as described in Fig. 1c.

for moderately increased affinities for hypoxanthine, guanine, uracil and xanthine analogues with bulky substitutions at positions 2, 3, 7 and 8, but not for analogues substituted at positions 1 and 9. For several mutants, this increase was smaller than that observed in the F528S genetic background. On the whole, however, affinities for purines other than uric acid and xanthine remain very low $(>700 \mu \mathrm{M})$, in agreement with the observation that all singlecopy plasmid mutants, besides A441V, could only grow well on adenine or hypoxanthine at $2 \mathrm{mM}$ (see Fig. 2). The only notable exception was mutant A441V, which showed a rather moderate affinity for hypoxanthine $(260 \mu \mathrm{M})$ that could in principle partially explain growth on $0.5 \mathrm{mM}$ hypoxanthine. However, the same mutant has no detectable affinity for adenine, despite its ability to grow on this purine at $0.5 \mathrm{mM}$, prompting us to believe that, even in that case, growth on purines other than uric acid or xanthine is due to increased capacity of transport per se, rather than increased substrate binding affinities (see later).

\section{Functional consequences of mutations affecting the TMS10-TMS11 loop}

To further investigate the role of the TMS10TMS11 loop and in particular that of residue R481, we followed a dual approach: First, we constructed double mutants by combining a selected mutation in R481 (R481G) with other mutations affecting UapA specificity, located in the TMS1-TMS2 loop (Q113L) or in TMS12 (T526M). Second, we made two UapA mutations that either delete R481 $(\Delta \mathrm{R} 481)$ or relocate its position within the TMS10TMS11 loop by introducing two small and flexible amino acid residues (Ala-Gly) just upstream from it (AGR481).

The double mutants Q113L/R481G and R481G/ T526M showed growth phenotypes very similar to R481G/F528S (i.e., they can grow on low adenine or hypoxanthine concentration; Fig. 3). This suggested that the effects of R481G and Q113L or T526M are additive, similar to R481G and F528S. The additive effect of R481G with all three previously described mutations affecting UapA specificity was also reflected in substrate binding affinities. Table 4 shows that the binding affinities for most purines of Q113L/R481G, and especially R481G/T526M, were significantly increased compared with the single mutants. More specifically, R481G/T526M shows high affinity for uracil $(32 \mu \mathrm{M})$, moderate affinity for hypoxanthine and guanine $(179-225 \mu \mathrm{M})$ and the highest affinity, albeit still low, for adenine $(\sim 1 \mathrm{mM})$, among all mutants described herein and previously. It also has increased affinity for xanthine, oxypurinol and nearly all xanthine analogues tested, with 9-methyl-xanthine being a notable exception.

The $\Delta$ R481 and AGR481 mutations inactivated UapA function, as the corresponding mutants could not grow in any purine as sole nitrogen source (Fig. 3a). To investigate the basis of phenotype, we analysed these mutants by making use of a GFP tag (addition of GFP at the C-terminus of UapA has no effect on UapA subcellular expression or on its function ${ }^{20}$ ). Figure $3 \mathrm{~b}$ shows that in both mutants, 
Table 3. Substrate binding profile of specificity mutants in the absence of F528S

\begin{tabular}{|c|c|c|c|c|c|c|c|c|c|}
\hline \multirow[b]{2}{*}{ Substrate/ligand } & \multicolumn{9}{|c|}{$K_{\mathrm{m} / \mathrm{i}}(\mu \mathrm{M})$} \\
\hline & $\mathrm{UapA}^{+}$ & Q113L & F406Y & A441V & V463I & A469E & $\mathrm{R} 481 \mathrm{G}$ & R481L & R481Q \\
\hline Xanthine & 8 & 4 & 3 & 4 & 1.3 & 8.5 & 18 & 3 & 0.9 \\
\hline Uric acid & 7 & 10 & 11 & 10 & 12 & 58 & 19 & 6 & 3.5 \\
\hline Adenine & NI & NI & NI & NI & $\sim 1700$ & $\sim 1500$ & NI & NI & $\sim 2500$ \\
\hline Hypoxanthine & NI & 260 & $\sim 2000$ & 260 & 900 & 880 & NI & NI & $\sim 1000$ \\
\hline Guanine & NI & $\sim 1000$ & $\sim 2000$ & $\mathrm{NI}$ & 750 & NI & NI & NI & $\sim 700$ \\
\hline Uracil & NI & 413 & $\sim 2000$ & 413 & 268 & 380 & $\sim 1000$ & NI & $\sim 1200$ \\
\hline 1-Methyl-xanthine & 280 & 280 & 420 & 280 & 300 & 500 & 200 & 150 & 200 \\
\hline 2-Thio-xanthine & 63 & 10 & 63 & 10 & 60 & 280 & 90 & 43.1 & 10.1 \\
\hline 3-Methyl-xanthine & 28 & 9 & 24 & 9 & 7 & 32.1 & 27 & 18.5 & 5.1 \\
\hline 6-Thio-xanthine & 350 & 38 & 92 & 38 & 132 & 300 & 56.6 & 59.1 & 3.7 \\
\hline 7-Methyl-xanthine & $\mathrm{NI}$ & $\sim 1000$ & $\sim 1000$ & $\sim 1000$ & 270 & 690 & 140 & 65.3 & 41.3 \\
\hline 8-Methyl-xanthine & 100 & 110 & 45 & 110 & 100 & 61 & 100 & 10 & 2.5 \\
\hline 9-Methyl-xanthine & 200 & NI & 345 & NI & 379 & NI & 124 & 286 & 200 \\
\hline Oxypurinol & 100 & 29 & 8.2 & 14.4 & 96.5 & 18.4 & 14.3 & 14.4 & 25.8 \\
\hline
\end{tabular}

For details, see Table 2.

UapA-GFP molecules label vacuolar lumens or other internal structures but not the plasma membrane-a picture characteristic of misfolded or extremely unstable transporters. The simplest explanation of this result is that altering the length of the TMS10-TMS11 loop destabilizes UapA.

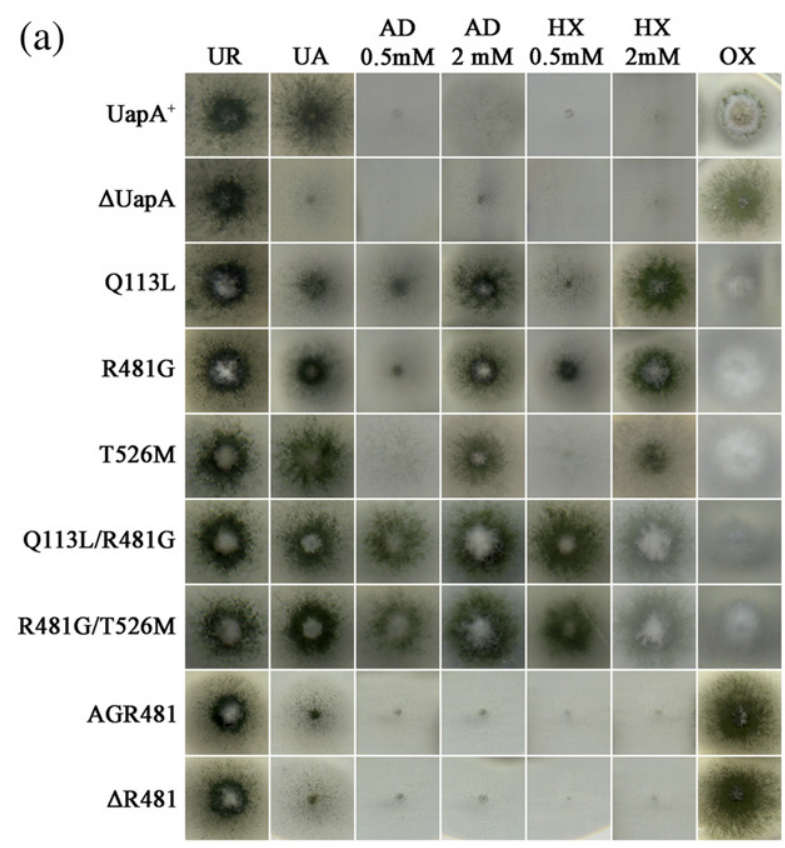

(b)
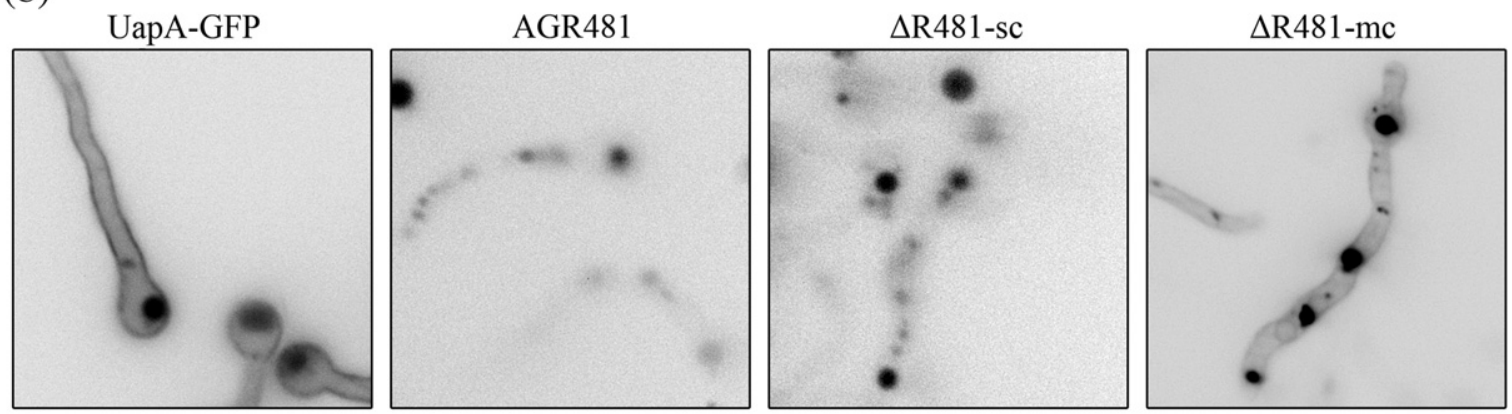

Fig. 3. (a) Growth tests of the constructed mutations on different purines as nitrogen sources. Control strains and growth conditions are described in Fig. 1b. (b) Epifluorescence microscopy of a wild-type (UapA-GFP) control and AGR481 and $\Delta$ R481 mutants. Strains were grown as described by Koukaki et al. ${ }^{7}$ In all mutant strains, UapA was weakly expressed in the plasma membrane and appeared mostly at the vacuoles. For $\Delta \mathrm{R} 481$, two strains are shown carrying a single copy $(\Delta \mathrm{R} 481-\mathrm{sc})$ or multiple copies $(\Delta \mathrm{R} 481-\mathrm{mc})$ of the mutated allele. All images have the same magnification. 
Table 4. Substrate binding profile of rationally designed double mutations

\begin{tabular}{|c|c|c|c|c|c|c|}
\hline \multirow[b]{2}{*}{ Substrate/ligand } & \multicolumn{6}{|c|}{$K_{\mathrm{m} / \mathrm{i}}(\mu \mathrm{M})$} \\
\hline & $\mathrm{UapA}^{+}$ & Q113L & R481G & $\mathrm{T} 526 \mathrm{M}^{\mathrm{a}}$ & Q113L/R481G & R481G/T526M \\
\hline Xanthine & 8 & 8 & 18 & 5 & 1.3 & 1.2 \\
\hline Uric acid & 7 & 7 & 19 & 12 & 2.5 & 8 \\
\hline Adenine & NI & NI & NI & $\sim 2500$ & NI & 980 \\
\hline Hypoxanthine & NI & NI & NI & $\sim 1700$ & 1300 & 179 \\
\hline Guanine & NI & NI & NI & ND & NI & 225 \\
\hline Uracil & NI & NI & $\sim 1000$ & $76 />1000$ & 536 & 32 \\
\hline 1-Methyl-xanthine & 280 & 280 & 200 & 288 & $\sim 300$ & $\sim 40$ \\
\hline 2-Thio-xanthine & 63 & 63 & 90 & 8 & 17 & 13 \\
\hline 3-Methyl-xanthine & 28 & 28 & 27 & 11 & 8 & 5.5 \\
\hline 6-Thio-xanthine & 350 & 350 & 57 & 6 & 4.5 & 4 \\
\hline 7-Methyl-xanthine & NI & NI & 140 & $\sim 1000$ & NI & 361 \\
\hline 8-Methyl-xanthine & 100 & 100 & 100 & 6 & 4 & 4 \\
\hline 9-Methyl-xanthine & 200 & 200 & 124 & 202 & NI & $\sim 1000$ \\
\hline Oxypurinol & 100 & 29 & 14 & 22 & 11 & 2 \\
\hline
\end{tabular}

For details, see Table 2. ND indicates not determined.

a Papageorgiou et al. ${ }^{10}$

\section{Discussion}

\section{UapA mutants with increased transport capacity for non-physiological substrates}

Our original goal was to design and test a direct genetic screen for obtaining UapA mutations conferring a specific increase in adenine affinity. Considering that adenine has a pyrimidine moiety very distinctive from that of other purines, we reasoned that such mutations would lead us to the identification of amino acid residues, within the UapA substrate binding pocket, that could directly interact with the so-called amidine group. Our results show that instead of isolating mutants with increased affinity specifically for adenine, we obtained mutations that increase the accumulation of purines other than the physiological substrates of UapA. As this increase concerns solely the transport of purines other than uric acid or xanthine, it cannot be due to an increase in the amount of permease present in the plasma membrane. This in turn suggests that the novel mutations increase the bona fide transport capacity of UapA specifically for purines that cannot be transported by the wild type or the F528S version of UapA. Importantly, despite a moderate increase in the binding of hypoxanthine or guanine conferred by mutations, all novel mutants still retain low affinities for purines $(>260 \mu \mathrm{M})$ other than uric acid or xanthine, independently of the presence of mutation F528S. Particularly for adenine, all mutants showed very low binding affinities $(>1.5 \mathrm{mM})$. This observation further supports the idea that the novel mutations lead to increased intrinsic transport capacity of UapA, specifically for purines other than uric acid or xanthine, rather than increased relative binding affinities. In that sense, the novel mutations are very similar to previously characterized substitutions located in TMS12 and in the TMS1TMS2 loop.

\section{Residues in TMS9 and TMS10 might line the substrate translocation pathway}

Unlike mutations concerning Q113, F406 and R481, which map in putative loops and have a pronounced effect on UapA transport specificity particularly in the presence of the F528S substitution, mutations A441V, V463I and A469E map in TMSs and seem to affect UapA transport specificity even in the absence of mutation F528S (compare growth on $0.5 \mathrm{mM}$ adenine in Figs. 1 and 2). This is evident in mutants arising from multiple-copy plasmid integrations, but at least for A441V, this is also apparent in single-copy transformants (see growth on $0.5 \mathrm{mM}$ adenine in Fig. 2). Furthermore, these mutations also lead to more drastic increases in the binding affinities of several nucleobases in the absence of mutation F528S, rather than in its presence (compare values in Tables 3 and 4). Despite an apparent additive effect of mutations in TMS10 (V463I or A469E) with F528S on the capacity of UapA to transport adenine or hypoxanthine, the effect of TMS9 or TMS10 mutations is not additive with that of F528S with respect to UapA specificity. These observations suggest that residues in TMS9 or TMS10 act independently to contribute in substrate specificity. Given their nature, we can speculate that the corresponding mutated residues contribute through specific van der Waals forces to the progressive sliding or fitting of purines within the substrate translocation pathway. Support for this comes from comparison with other NATs of known specificities. The residue corresponding to V463 in other NAT members is either a Val or an Ile, which, together with the mutation isolated in UapA (V463I), shows that these amino acids might be the only functionally permissive residues at this position. Residue A469 is more variable, being replaced in other NATs by Arg, Ser or Lys. Interestingly, NAT homologues with no (e.g., bacterial members Ygfo, YicE and PbuX) or moderate (e.g., UapC of $A$. nidulans and Aspergillus fumigatus or Xut1 of Candida 
albicans) affinity for uric acid do not possess an Ala residue. The possible contribution of Ala469 in uric acid binding in UapA is supported by the fact that mutation A469E significantly affects (8.5-fold reduction) uric acid binding (see Table 3). Finally, A441 is relatively well conserved, although some NAT members have other small amino acid residues at this position (Gly, Ser, Thr, Pro). The role of the above residues on UapA function could not have been predicted by a rational design of mutations.

\section{Synergy of loops TMS1-TMS2, TMS8-TMS9 and TMS12 determines UapA specificity}

We have previously provided evidence that very fine interactions between the TMS8-TMS9 loop, hosting basic elements of the substrate translocation pathway, and the TMS1-TMS2 loop or TMS12, two regions affecting substrate specificity, define the function of UapA, particularly with respect to substrate selection and binding affinity. Here, we obtained new unbiased evidence that TMS12 interacts functionally with the TMS8-TMS9 and TMS1TMS2 loops. Mutations Q113L (TMS1-TMS2 loop) and F406Y (in the NAT motif in TMS8-TMS9 loop) promote increased transport capacity for adenine, hypoxanthine and probably other several nucleobases only when combined with F528S (TMS12). The additive phenotypes of these mutations are also reflected in differences in the $K_{\mathrm{m} / \mathrm{i}}$ values for several nucleobases (e.g., xanthine, uric acid, guanine, uracil) and nucleobase analogues. These results confirm that substitutions in these three domains additively inactivate the synergistic interactions responsible for the high substrate specificity of UapA.

\section{Dynamic elements at both sides of UapA selectively control the accessibility of sub- strates to their translocation pathway}

The great majority of mutants (20 of $28, \sim 72 \%$ ) selected in the background of F528S concerned residue R481, located in the short putative cytoplasmic loop that links TMS10 with TMS11. This arginine residue is conserved only in fungal homologues, whereas in bacterial, plant and animal NAT members, it is replaced by Leu, Ile, Thr or Lys. Interestingly, in the homologous mammalian NATs, the residues corresponding to UapA R481 are leucines, irrespective of whether they are specific for ascorbate (SVCT1 and SVCT2 $)^{2,13}$ or nucleobases (rSNBT) ${ }^{14}$ However, none of the mutations of R481, including R481L, conferred to UapA a detectable binding affinity for ascorbate (data not shown). The lack of evolutionary conservation at this position is in line with the observation that mutants with variable substitutions all proved functional. This suggests that R481 might be involved in a fine topological interaction specifically narrowing the selectivity of UapA. Positive cytoplasm-facing residues are believed to play important topological roles in several membrane proteins through their interaction with negatively charged lipids of the inner leaflet of the plasma membrane. ${ }^{21}$ Alternatively, R481 might interact with a negatively charged residue of UapA and thus create a specific bridge that blocks the passage of purines other than uric acid or xanthine. Interestingly, mutations that delete R481 or affect the length, and apparently the flexibility, of the TMS10-TMS11 loop lead to unstable non-functional UapA molecules that are rapidly turned over. In any way, this short loop seems to act as a key element in the dynamic positioning of the TMS10 and TMS11 $\alpha$-helices and thus for the selection and translocation of specific substrates through UapA.

The putative inward-facing topology of R481 and the additive nature of R481 substitutions with mutations located toward the extracytoplasmic face of the transporter, which also reduce specificity, strongly suggest that UapA has molecular determinants acting as selective gates at both sides of the plasma membrane. Gating is a term used mostly for channels and usually implies the dynamic action of domains or some amino acid residues, triggered through either ligand binding or a change in voltage, which allows or restricts the passage of ions through a rather rigid and continuous transmembrane pore. For transporters, the dogma is that they do not form a continuous pore and thus do not use a gating mechanism. They rather exist in at least two alternating conformations, making them accessible to substrates either intracellularly or extracellularly, through a rocker-switch mechanism. In fact, it is probably the binding of substrates that allosterically promotes the alternation of the different conformers. In the last 2 years, however, a number of crystal structures of evolutionary and functionally distinct transporters confirmed the existence of domains or loops at both sides of the plasma membrane that occlude or open the access of substrates to the sensu stricto binding site. ${ }^{22-28}$ Our results provide strong functional evidence for the existence of such dynamic domains or amino acids, distinct from the substrate binding site, that are implicated in subtle movements determining the function and specificity of a transporter.

\section{Materials and Methods}

\section{Media, strains, growth conditions and transformation genetics}

Standard media for $A$. nidulans and $E$. coli were used. ${ }^{29}$ The $\Delta$ uapA $\Delta$ uapC $\Delta a z g A \Delta f c y B$ pabaA1 strain ( $\Delta$ UapA) carrying a single-copy integration of the uap $A\left(\mathrm{UapA}^{+}\right)$was a standard wild-type control. The strain used to select the original mutants was a $\Delta u a p A \Delta u a p C \Delta a z g A \Delta f c y B$ pabaA1 $(\triangle \mathrm{UapA})$ carrying a single-copy integration of the uapAF528S (F528S). ${ }^{10}$ pabaA1 is a genetic auxotrophy for $p$ aminobenzoic acid. UV mutagenesis was as described previously. ${ }^{6}$ An isogenic $\Delta$ uapA $\Delta$ uapC $\Delta a z g A \quad \Delta f c y B$ pabaA1 mutant was the recipient strain in transformations ${ }^{30}$ with uap $A$ alleles cloned in vector pAN520 $\exp ^{31}$ or with an 'empty' vector as a negative control. These vectors allow selection of transformants based on $p$-aminobenzoic acid auxotrophy complementation. Transformants expressing intact uapA or uapA-gfp alleles, through either single- or 
multiple-copy plasmid integration events, were identified by PCR and Southern analysis. In most cases, we selected transformants originating from homologous integration in the paba $A$ locus. In some cases, single-copy uap $A$ alleles were integrated heterologously, but these transformants expressed UapA also at physiological levels (not shown). Growth tests were performed at 25 and $37{ }^{\circ} \mathrm{C}$ and at different purine concentrations $(0.5$ and $2 \mathrm{mM}): 0.5 \mathrm{mM}$ is the standard purine concentration used in all previous studies concerning purine metabolism in A. nidulans. Oxypurinol was used at $100 \mu \mathrm{M}$, with nitrate $(10 \mathrm{mM})$ as a nitrogen source. uapA alleles resulted in phenotypes that were independent of the presence or absence of the Cterminal $g f p$ epitope. ${ }^{20}$

\section{Plasmid constructions and uapA mutations}

pAN510 is a pBlueScript vector carrying a uapA fusion with its flanking sequences and the $\arg B$ gene as a selection marker. ${ }^{20}$ pAN510-GFP is a pBlueScript vector carrying a uapA-gfp fusion with its flanking sequences and the $\arg B$ gene as a selection marker. ${ }^{20}$ pAN510exp is a modified version of pAN510, introducing a BamHI site at the translation start codon and knocking out an $\mathrm{XbaI}$ site in the pBlueScript multicloning region for cloning purposes. ${ }^{19}$ pAN520exp is a modified version of pAN510exp, replacing the auxotrophic marker $\arg B$ with $p a b a A$ at the Sall site of the plasmid. The uapA ORF from the original mutants was amplified by PCR using primers $5^{\prime}$-cgggatccctccatccattcaaccgac- $3^{\prime}$ and $5^{\prime}$-gctctagactaagctgcttgctctgatac- $3^{\prime}$, cloned in pAN510exp and sequenced + . Mutations $\Delta R 481$ and AGR481 were constructed by site-directed mutagenesis according to the QuikChange ${ }^{\circledR}$ Site-Directed Mutagenesis Kit (Stratagene) on vector pAN510-GFP using complementary oligonucleotides carrying the desired substitution (forward primers: 5'-ccgttcacaaggcgaaatgccggccggtttatcctcaccgcg- $3^{\prime}$ and $5^{\prime}$-gcgccgttcacaaggcgaaattttatcctcaccgcgtcaatg- $3^{\prime}$, respectively) and confirmed by sequencing. The uapA-gfp fusion was amplified by PCR, using primers $5^{\prime}$ cgggatccctccatccattcaaccgac- $3^{\prime}$ and $5^{\prime}$-cgtctagattacttgtacagctcgtcc-3', and subsequently cloned in pAN520exp. Finally, the constructed plasmids were introduced by transformation into a $\Delta$ uapA $\Delta u a p C \Delta a z g A \Delta f c y B$ pabaA1 strain, and the resulting integration events were subjected to Southern analysis. ${ }^{32}$ Single mutations F406Y, A441V, V463I, A469E, R481Q, R481L and R481G were constructed by replacing the SacI/SacI fragment from each double mutant with the corresponding fragment of pAN510exp. Q113L/ $\mathrm{R} 481 \mathrm{G}$ was constructed by replacing the $\mathrm{ClaI} / \mathrm{XbaI}$ fragment of pAN510expUapA-R481G with the corresponding fragment from pAN510expUapA-Q113L, containing the Q113L mutation. R481G/T526M was constructed by replacing the SacI/SacI fragment of pAN510expUapA-T526M with the corresponding fragment from pAN510expUapA-R481G, containing the R481G mutation. In all cases, the uapA ORF was amplified by PCR using the primers $5^{\prime}$-cgggatccctccatccattcaaccgac- $3^{\prime}$ and $5^{\prime}$-gctctagactaagcetgcttgctctgatac-3 and subsequently cloned in pAN520exp.

\section{Standard nucleic acid manipulations and fluorescence microscopy}

Southern blot and PCR analyses were performed as described by Sambrook et al. ${ }^{32}\left[{ }^{32} \mathrm{P}\right] \mathrm{dCTP}$-labelled molecules used as pabaA- or uapA-specific probes were prepared

$\dagger$ http:/ / www.macrogen.com using a random hexanucleotide primer kit following the supplier's instructions (Takara, Japan). Labelled [ $\left.{ }^{32} \mathrm{P}\right] \mathrm{dCTP}$ (3000 Ci/mmol) was purchased from the Institute of Isotopes Co., Ltd. Total genomic DNA isolation from $A$. nidulans strains and all other techniques involving DNA manipulations were described by Sambrook et al. ${ }^{32}$ Samples for fluorescence microscopy were prepared as described previously. ${ }^{20}$ In brief, the samples were incubated on coverslips in liquid minimal medium ${ }^{29}$ supplemented with urea as nitrogen source at $25^{\circ} \mathrm{C}$ for $12-14 \mathrm{~h}$, observed on an Axioplan Zeiss phase-contrast epifluorescence microscope with appropriate filters, and the resulting images were acquired with a Zeiss MRC5 digital camera using the AxioVs40 V4.40.0 software. Images were then processed in Adobe Photoshop CS2 V9.0.2 software.

\section{Kinetic analysis}

Radiolabelled purines (19.6-33.4 Ci/mmol) were from Moravek Biochemicals (Brea, CA). ${ }^{3} \mathrm{H}$-labelled xanthine or hypoxanthine uptake was assayed in conidiospores at 37 or $25^{\circ} \mathrm{C}$ as described previously. ${ }^{5}$ All experiments were carried out in triplicate. Initial velocities were corrected by subtracting background uptake values, measured in the azg $A^{-}$uap $A^{-}$uap $C^{-} f c y B^{-}$mutant. $K_{\mathrm{m}}$ (concentration for obtaining $V_{\mathrm{m}} / 2$ ) and $\mathrm{IC}_{50}$ (concentration reducing $50 \%$ of the $V_{\mathrm{m}}$ ) values for compounds inhibiting the uptake of radiolabelled permeants (xanthine or hypoxanthine) were determined from full dose-response curves with a minimum of eight points spread over the relevant range. In all cases, the Hill coefficients were close to -1 , consistent with competitive inhibition. $K_{\mathrm{i}}$ values were calculated from the Cheng and Prusoff equation, $K_{\mathrm{i}}=\mathrm{IC}_{50} /$ $\left[1+\left(L / K_{\mathrm{m}}\right)\right]$, in which $L$ is the permeant concentration. ${ }^{33}$

\section{Speculative UapA topology}

Speculative UapA topology was based on several standard algorithms (PredictProtein, HHpred, TMHMM, SOSUI, TMAP, TopPred, etc.), the ConSeq program $\ddagger$ and minor manual modifications depending on the presence of Pro residues and the 'positive-inside rule ${ }^{\prime 21}$ at the borders of putative helices. All algorithms predict as $\alpha$-helical transmembrane domains the segments shown in Fig. 1 as TMS1TMS8 and TMS12. Most algorithms also predict the TMS9bTMS11 shown in Fig. 1. Some algorithms predict an extra TMS, which here we call TMS9a (horizontal cylinder in Fig. 1 ), in the segment between TMS8 and TMS9b. Based on the above and the substituted-cysteine accessibility method performed in $\mathrm{YgfO}$, which shows that TMS9a has extended parts accessible to hydrophilic reagents (see the text), we propose that this segment is a flexible dynamic domain or a reentrant loop that acquires alternative topology during substrate binding and transport.

\section{Acknowledgements}

Work in the laboratory of G.D. was partly supported by the University of Athens (ELKE). We are grateful to $\mathrm{S}$. Amillis for helping in the preparation of the manuscript and for discussions. 


\section{References}

1. Gorfinkiel, L., Diallinas, G. \& Scazzocchio, C. (1993). Sequence and regulation of the uap $A$ gene encoding a uric acid-xanthine permease in the fungus Aspergillus nidulans. J. Biol. Chem. 268, 23376-23381.

2. Gournas, C., Papageorgiou, I. \& Diallinas, G. (2008). The nucleobase-ascorbate transporter (NAT) family: genomics, evolution, structure-function relationships and physiological role. Mol. BioSyst. 4, 404-416.

3. Diallinas, G. \& Gournas, C. (2008). Structure-function relationships in the nucleobase-ascorbate transporter (NAT) family: lessons from model microbial genetic systems. Channels (Austin), 2, 363-372.

4. Diallinas, G., Valdez, J., Sophianopoulou, V., Rosa, A. \& Scazzocchio, C. (1998). Chimeric purine transporters of Aspergillus nidulans define a domain critical for function and specificity conserved in bacterial, plant and metazoan homologues. EMBO J. 17, 3827-3837.

5. Meintanis, C., Karagouni, A. D. \& Diallinas, G. (2000). Amino acid residues N450 and Q449 are critical for the uptake capacity and specificity of UapA, a prototype of a nucleobase-ascorbate transporter family. Mol. Membr. Biol. 17, 47-57.

6. Amillis, S., Koukaki, M. \& Diallinas, G. (2001). Substitution F569S converts UapA, a specific uric acid-xanthine transporter, into a broad specificity transporter for purine-related solutes. J. Mol. Biol. 313, 765-774.

7. Koukaki, M., Vlanti, A., Goudela, S., Pantazopoulou, A., Gioule, H., Tournaviti, S. \& Diallinas, G. (2005). The nucleobase-ascorbate transporter (NAT) signature motif in UapA defines the function of the purine translocation pathway. J. Mol. Biol. 350, 499-513.

8. Pantazopoulou, A. \& Diallinas, G. (2006). The first transmembrane segment (TMS1) of UapA contains determinants necessary for expression in the plasma membrane and purine transport. Mol. Membr. Biol. 23, 337-348.

9. Vlanti, A., Amillis, S., Koukaki, M. \& Diallinas, G. (2006). A novel-type substrate-selectivity filter and ER-exit determinants in the UapA purine transporter. J. Mol. Biol. 357, 808-819.

10. Papageorgiou, I., Gournas, C., Vlanti, A., Amillis, S., Pantazopoulou, A. \& Diallinas, G. (2008). Specific interdomain synergy in the UapA transporter determines its unique specificity for uric acid among NAT carriers. J. Mol. Biol. 382, 1121-1135.

11. Goudela, S., Karatza, P., Koukaki, M., Frillingos, S. \& Diallinas, G. (2005). Comparative substrate recognition by bacterial and fungal purine transporters of the NAT/NCS2 family. Mol. Membr. Biol. 22, 263-275.

12. Argyrou, E., Sophianopoulou, V., Schultes, N. \& Diallinas, G. (2001). Functional characterization of a maize purine transporter by expression in Aspergillus nidulans. Plant Cell, 13, 953-964.

13. Tsukaguchi, H., Tokui, T., Mackenzie, B., Berger, U. V., Chen, X. Z., Wang, Y. et al. (1999). A family of mammalian $\mathrm{Na}^{+}$-dependent L-ascorbic acid transporters. Nature, 399, 70-75.

14. Yamamoto, S., Inoue, K., Murata, T., Kamigaso, S., Yasujima, T., Maeda, J. Y., et al. (in press). Identification and functional characterization of the first nucleobase transporter in mammals: implication in the species difference in the intestinal absorption mechanism of nucleobases and their analogs between higher primates and other mammals J. Biol. Chem., doi/10.1074/jbc.M109.032961.
15. Karatza, P. \& Frillingos, S. (2005). Cloning and functional characterization of two bacterial members of the NAT/NCS2 family in Escherichia coli. Mol. Membr. Biol. 22, 251-261.

16. Karatza, P., Panos, P., Georgopoulou, E. \& Frillingos, S. (2006). Cysteine-scanning analysis of the nucleobase-ascorbate transporter signature motif in $\mathrm{YgfO}$ permease of Escherichia coli: Gln-324 and Asn-325 are essential, and Ile-329-Val-339 form an alpha-helix. J. Biol. Chem. 281, 39881-39890.

17. Papakostas, K., Georgopoulou, E. \& Frillingos, S. (2008). Cysteine-scanning analysis of putative helix XII in the YgfO xanthine permease: ILE-432 and ASN-430 are important. J. Biol. Chem. 283, 13666-13678.

18. Karena, E. \& Frillingos, S. (2009). Role of intramembrane polar residues in the YgfO xanthine permease: HIS-31 and ASN-93 are crucial for affinity and specificity, and ASP-304 and GLU-272 are irreplaceable. J. Biol. Chem. 284, 24257-24268.

19. Vlanti, A. \& Diallinas, G. (2008). The Aspergillus nidulans FcyB cytosine-purine scavenger is highly expressed during germination and in reproductive compartments and is downregulated by endocytosis. Mol. Microbiol. 68, 959-977.

20. Pantazopoulou, A., Lemuh, N. D., Hatzinikolaou, D. G., Drevet, C., Cecchetto, G., Scazzocchio, C. \& Diallinas, G. (2007). Differential physiological and developmental expression of the UapA and AzgA purine transporters in Aspergillus nidulans. Fungal Genet. Biol. 44, 627-640.

21. von Heijne, G. \& Gavel, Y. (1988). Topogenic signals in integral membrane proteins. Eur. J. Biochem. 174, 671-678.

22. Yamashita, A., Singh, S. K., Kawate, T., Jin, Y. \& Gouaux, E. (2005). Crystal structure of a bacterial homologue of $\mathrm{Na}^{+} / \mathrm{Cl}^{-}$-dependent neurotransmitter transporters. Nature, 437, 215-223.

23. Faham, S., Watanabe, A., Besserer, G. M., Cascio, D., Specht, A., Hirayama, B. A. et al. (2008). The crystal structure of a sodium galactose transporter reveals mechanistic insights into $\mathrm{Na}^{+}$/ sugar symport. Science, 321, 810-814.

24. Weyand, S., Shimamura, T., Yajima, S., Suzuki, S., Mirza, O., Krusong, K. et al. (2008). Structure and molecular mechanism of a nucleobase-cationsymport-1 family transporter. Science, 322, 709-713.

25. Singh, S. K., Piscitelli, C. L., Yamashita, A. \& Gouaux, E. (2008). Antidepressant binding site in a bacterial homologue of neurotransmitter transporters. Science, $322,1655-1661$.

26. Diallinas, G. (2008). Biochemistry. An almost-complete movie. Science, 322, 1644-1645.

27. Fang, Y., Jayaram, H., Shane, T., KolmakovaPartensky, L., Wu, F., Williams, C. et al. (2009). Structure of a prokaryotic virtual proton pump at $3.2 \AA$ resolution. Nature, 460, 1040-1043.

28. Gao, X., Lu, F., Zhou, L., Dang, S., Sun, L., Li, X. et al. (2009). Structure and mechanism of an amino acid antiporter. Science, 324, 1565-1568.

29. Cove, D. J. (1966). The induction and repression of nitrate reductase in the fungus Aspergillus nidulans. Biochim. Biophys. Acta, 113, 51-56.

30. Koukaki, M., Giannoutsou, E., Karagouni, A. \& Diallinas, G. (2003). A novel improved method for Aspergillus nidulans transformation. J. Microbiol. Methods, 55, 687-695.

31. Gournas, C., Amillis, S., Vlanti, A. \& Diallinas, G. (2009). Transport-dependent endocytosis and 
turnover of a uric acid-xanthine permease. Mol. Microbiol. 75, 246-260.

32. Sambrook, J., Fritsch, E. \& Maniatis, T. (1989). Molecular Cloning: A Laboratory Manual. Cold Spring Harbor Press, Cold Spring Harbor, NY.
33. Cheng, Y. \& Prusoff, W. H. (1973). Relationship between the inhibition constant (K1) and the concentration of inhibitor which causes 50 per cent inhibition (I50) of an enzymatic reaction. Biochem. Pharmacol. 22, 3099-3108. 